\title{
AN5D: Automated Stencil Framework for High-Degree Temporal Blocking on GPUs
}

\author{
Kazuaki Matsumura* \\ Barcelona Supercomputing Center, Spain \\ kazuaki.matsumura@bsc.es
}

\author{
Hamid Reza Zohouri* \\ Edgecortix Inc., Japan \\ hamid@edgecortix.com
}

\author{
Mohamed Wahib \\ AIST, Japan \\ mohamed.attia@aist.go.jp
}

\author{
Toshio Endo \\ Tokyo Institute of Technology, Japan \\ endo@is.titech.ac.jp
}

\author{
Satoshi Matsuoka \\ RIKEN CCS, Japan \\ matsu@acm.org
}

\begin{abstract}
Stencil computation is one of the most widely-used compute patterns in high performance computing applications. Spatial and temporal blocking have been proposed to overcome the memory-bound nature of this type of computation by moving memory pressure from external memory to on-chip memory on GPUs. However, correctly implementing those optimizations while considering the complexity of the architecture and memory hierarchy of GPUs to achieve high performance is difficult. We propose AN5D, an automated stencil framework which is capable of automatically transforming and optimizing stencil patterns in a given $\mathrm{C}$ source code, and generating corresponding CUDA code. Parameter tuning in our framework is guided by our performance model. Our novel optimization strategy reduces shared memory and register pressure in comparison to existing implementations, allowing performance scaling up to a temporal blocking degree of 10 . We achieve the highest performance reported so far for all evaluated stencil benchmarks on the state-of-the-art Tesla V100 GPU.
\end{abstract}

CCS Concepts - Software and its engineering $\rightarrow$ Source code generation.

Keywords Stencil Computation, GPU, Automatic Code Generation, Temporal Blocking

\section{ACM Reference Format:}

Kazuaki Matsumura, Hamid Reza Zohouri, Mohamed Wahib, Toshio Endo, and Satoshi Matsuoka. 2020. AN5D: Automated Stencil Framework for High-Degree Temporal Blocking on GPUs. In Proceedings of the 18th ACM/IEEE International Symposium on

Permission to make digital or hard copies of all or part of this work for personal or classroom use is granted without fee provided that copies are not made or distributed for profit or commercial advantage and that copies bear this notice and the full citation on the first page. Copyrights for components of this work owned by others than ACM must be honored. Abstracting with credit is permitted. To copy otherwise, or republish, to post on servers or to redistribute to lists, requires prior specific permission and/or a fee. Request permissions from permissions@acm.org.

CGO '20, February 22-26, 2020, San Diego, CA, USA

(c) 2020 Association for Computing Machinery.

ACM ISBN 978-1-4503-7047-9/20/02 ..\$15.00

https://doi.org/10.1145/3368826.3377904
Code Generation and Optimization (CGO '20), February 22-26, 2020, San Diego, CA, USA. ACM, New York, NY, USA, 13 pages. https://doi.org/10.1145/3368826.3377904

\section{Introduction}

Stencil computation is one of the most frequently used computation patterns in High Performance Computing (HPC) that is often highly iterative [1, 28-30]. Despite non-stop advancements in both hardware and compiler technologies, optimizing this computation pattern on modern hardware remains a challenge due to its memory-bound nature. Currently, GPUs are the most popular accelerator in supercomputers and are employed in half of the top 10 machines in the TOP500 June 2019 list [33]. Although the computational performance of GPUs has been increasing rapidly, the gap between their computational performance and memory bandwidth prevents full utilization of their computational performance for HPC applications that rely on stencil computation. Temporal blocking $[9,12,16-18,20,21,23,24,26]$ is a well-known technique proposed to relieve memory pressure in stencil computation by combining multiple consecutive iterations of the time loop and avoiding the memory transactions required between them. However, correct and efficient implementation of this technique is challenging since it requires careful management of limited register and shared memory resources on GPUs. As a result, most existing work that implement temporal blocking is limited to low degrees of temporal blocking. Moreover, such work is generally implemented manually on a limited set of benchmarks $[17,20]$, or through frameworks that are not available for public use [23, 24, 26]. In this work, we present our open-source high-performance stencil code generation framework called AN5D ['ændi] (Auto N.5D). Our framework accepts unoptimized stencil patterns in C language, implements spatial and temporal blocking alongside with multiple low-level optimizations, and generates associated CUDA host and kernel code. Our contributions are as follows:

*This work was performed while those authors were at Tokyo Institute of Technology and K. Matsumura was a research assistant at RWBC-OIL. 
- We create an automated stencil framework to automatically implement spatial and temporal blocking from a C-based stencil description and automatically generate associated CUDA code.

- Through substantial engineering effort, we implement multiple low-level optimization techniques such as associative stencil optimization, shared memory double-buffering to reduce shared memory usage, and data movement reduction to optimize register usage, all in a cohesive fashion. This allows us to scale performance with temporal blocking degrees up to 10 on GPUs, which had never been achieved before.

- We perform a comprehensive comparison with state-ofthe-art implementations of stencil computation on GPUs (Hybrid Tiling and deep temporal tiling) and show that our framework achieves the highest performance reported so far for a wide range of stencil shapes and orders on the latest NVIDIA GPUs.

- We make our framework available to public to relieve the community from the burden of having to implement all those optimizations manually.

\section{Background}

\subsection{Stencil Computation}

In stencil computation, grid cells from a multi-dimensional input are iteratively updated based on a specific computation pattern; this pattern is defined by the stencil shape. The most widely used stencil shapes are star and box. Star stencils involve accessing neighbors that differ from the current cell only in the direction of one dimension at a time (i.e. no diagonal accesses), while box stencils form full squares (for 2D) or cubes (for 3D). The computation pattern involves neighboring accesses along each dimension up to a certain distance called the stencil radius. We call a stencil with radius of rad a "rad $d^{\text {th }}$-order stencil" . The calculation generally involves a sum of products over the values of the current cell and its neighbors and a set of coefficients that might or might not be shared among these cells. Therefore, each calculation depends on the calculation of neighbors in the previous iteration (also called time-step).

\subsection{Spatial Blocking}

Since there is no data dependency within the same time-step for explicit solvers, the spatial order of stencil updates within one time-step can be arbitrary. Spatial blocking $[13,38]$ splits the input grid into multiple blocks (or sub-planes) to accelerate execution by increasing the locality. On GPUs, rather than purely relying on the cache hierarchy, on-chip resources can be used to explicitly implement spatial blocking. After one block of data is moved from global memory to on-chip memory, the

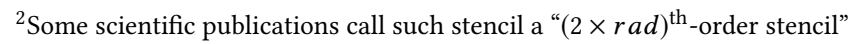

remaining loads in the time-step from the same block are done with no global memory access. However, loading boarder neighbor cells which belong to adjacent blocks will result in redundant global memory accesses.

\subsection{Temporal Blocking}

Even though stencil computation has data dependency across time-steps, the dependency range of one cell is limited by the product of the stencil radius ( $\mathrm{rad}$ ) and the number of time-steps passed since the cell's last update $\left(b_{\mathrm{T}}\right)$. Temporal blocking exploits the hidden temporal locality by combining multiple consecutive time-steps and avoiding global memory accesses in between. The dependency along the time dimension is resolved by redundantly loading and computing cells from adjacent blocks, the amount of which will increase with $\mathrm{rad}$ and $b_{\mathrm{T}}$.

Overlapped tiling $[12,16,18,23]$ is a form of temporal blocking which involves overlapping the spatial blocks by $2 \times b_{\mathrm{T}} \times \mathrm{rad}$ rows and columns (called halo regions) and redundantly loading and computing necessary cells that would fall inside surrounding blocks to process $b_{\mathrm{T}}$ time-steps with only one round of global memory loads and stores per block. Overlapped tiling is also applicable over spatial blocking methods other than domain decomposition (i.e. blocking all the input dimensions). 3.5D blocking, which implements 1D overlapped tiling (2 combined time-steps) on top of 2.5D spatial blocking for 3D stencils, was introduced in [20]. 2.5D spatial blocking involves blocking two dimensions and streaming the computation over the third one. Similarly, 1.5D spatial blocking can be used for 2D stencils. This blocking technique has been widely employed on different devices [17, 23, 40, 41], with not just two, but also more combined time-steps. In 2.5D spatial blocking, the 2D tiles are streamed over one dimension and data of each tile is effectively reused for updating the next tiles. To minimize the use of on-chip memory, the computational flow forms tiers along time-steps as shown in Fig. 1; here, each tile is evicted from on-chip memory as soon as no computation depends on its data anymore, and is replaced by a new tile. 3.5D blocking can be extended to support any arbitrary number of combined time-steps; we call this N.5D blocking.

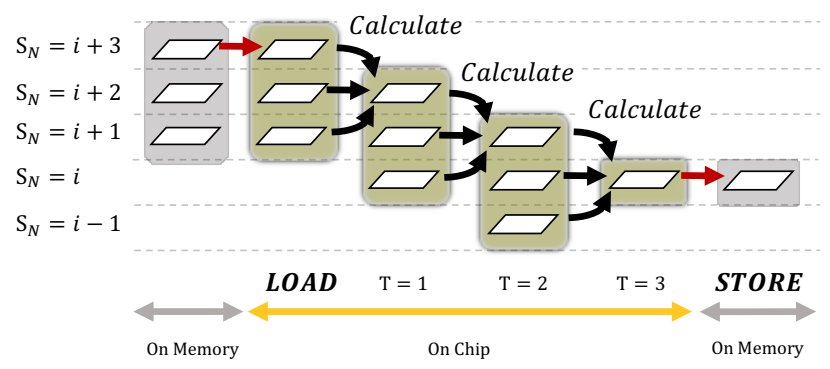

Figure 1. Computational flow of N.5D blocking with temporal blocking size of 3 and stencil radius of 1 
In contrast to overlapped tiling, non-redundant temporal blocking techniques [2, 8, 14, 19] realize temporal blocking without introducing redundant computation. To resolve the temporal dependency, such tiling methods form geometric (e.g. trapezoidal, wavefront) blocks along the time dimension. However, the dependency between neighboring blocks restricts parallelism in those methods.

\section{Related Work}

In [24, 26], Rawat et al. present a DSL-based stencil framework named STENCILGEN that implements N.5D blocking with several optimizations including DAG fusion. In this framework, shared memory is used for accessing nearby cells from each thread within a block, and one shared memory buffer is required per combined time-step. For diagonal-access free (a.k.a star) stencils, registers are used to keep the previous time-step's results of both the upper and the lower sub-planes. For other stencil types, if the stencil is associative, the computation of each cell is carried out in multiple steps, with each step only performing the parts of the computation that access one sub-plane (partial summation). This way, it is not required to keep all sub-planes that need to be accessed for computation of a cell in shared memory at the same time and instead, sub-planes are computed one by one and the result of the partial sum is stored in a register. Each partial sum is then used for computing the next sub-plane to complete the summation. This technique was also used in [31] to improve global memory access alignment and efficiency. Moreover, STENCILGEN supports the division of the streaming dimension for increasing thread-block-level parallelism, at the cost of additional redundant computations along the streaming dimension. Main advantages of our framework over STENCILGEN are: 1) Our framework uses standard C code as input while STENCILGEN is DSL-based. 2) Our framework is generic and publicly available (some benchmarks optimized by STENCILGEN are publicly available but not the framework itself). 3) Our framework reduces both shared memory and register usage by reducing register movement and employing shared memory double-buffering instead of multi-buffering (Section 4.1 and Table 1), 4) And most importantly, our novel optimization strategy allows performance scaling up to two-digit degrees of temporal blocking, while STENCILGEN's performance scaling is limited to a degree of 4 [24, 26]. In [27], Rawat et al. present another DSL-based stencil framework called ARTEMIS which supports flexible resource allocation on GPUs (global memory or share memory + register) for each input/output grid especially for high-order multi-array and multi-statement stencils. In [25], the same authors present a statement reordering framework for complex high-order stencils that avoids using shared memory and minimizes register pressure by using a register scheduling algorithm to

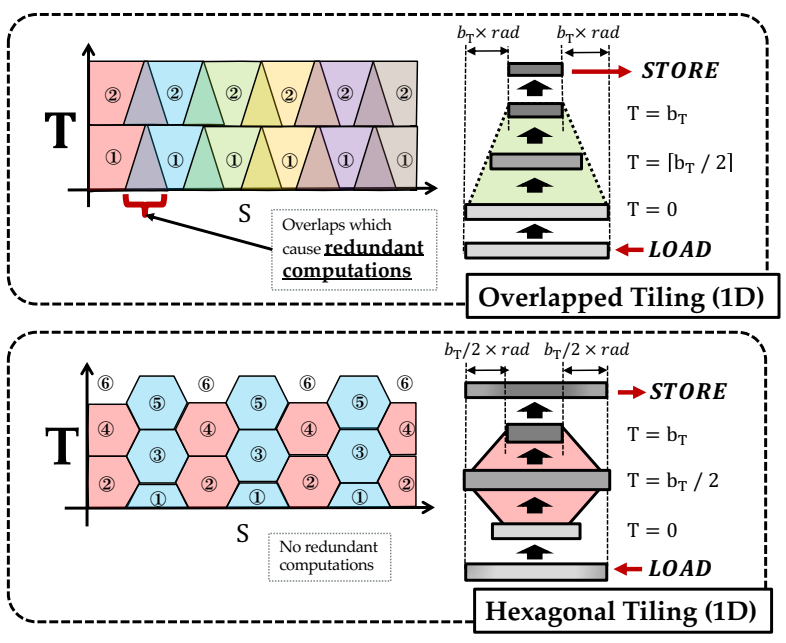

Figure 2. Computational flow of 1D overlapped and hexagonal tiling. Red arrows indicate global memory I/O and black arrows depict the update flow that involves on-chip memory accesses.

re-arrange the sequence of memory access/compute statements. In contrast, AN5D focuses on low-level optimizations to enable high-degree temporal blocking that could complement such optimizations.

Hybrid tiling [7, 9, 10, 21] is a prominent method for non-redundant temporal blocking which combines hexagonal tiling and classical wavefront tiling. The computational flow of hexagonal tiling is shown in Fig. 2. This schedule ( (1) $\rightarrow$ (2) $\rightarrow$ (3) $\rightarrow$... ) allows discrete blocks to be executed in parallel while the hexagonal shape allows resolving the temporal dependency without redundant computation. Apart from the time dimension, hybrid tiling employs hexagonal tiling only for one of the spatial dimensions, while remaining dimensions are blocked in a wavefront manner. The main shortcoming of this technique is that it blocks all spatial dimensions (no streaming) and hence, is limited to smaller block sizes compared to N.5D blocking for the same amount of on-chip memory. This leads to higher ratio of redundant-to-valid memory accesses and lower scaling with temporal blocking compared to

Table 1. Comparison to STENCILGEN

\begin{tabular}{|c|c|c|}
\hline & STENCILGEN [26] & AN5D \\
\hline Register Allocation & Shifting & Fixed \\
\hline Shared Memory Use & For Streaming & For Calculation \\
\hline \multicolumn{3}{|c|}{ Shared Memory Footprint Per Block: } \\
\hline Diagonal-Access Free & $n_{\mathrm{thr}} \times b_{\mathrm{T}} \times n_{\text {word }}$ & $2 \times n_{\mathrm{thr}} \times n_{\text {word }}$ \\
\hline Associative Stencil & $n_{\mathrm{thr}} \times b_{\mathrm{T}} \times n_{\text {word }}$ & $2 \times n_{\mathrm{thr}} \times n_{\mathrm{word}}$ \\
\hline \multirow{2}{*}{ Otherwise } & $n_{\mathrm{thr}} \times b_{\mathrm{T}} \times$ & $2 \times n_{\mathrm{thr}} \times$ \\
\hline & $(1+2 \times r a d) \times n_{\text {word }}$ & $(1+2 \times r a d) \times n_{\text {word }}$ \\
\hline \multicolumn{3}{|c|}{ Shared Memory Store Per Cell: } \\
\hline Diagonal-Access Free & 1 & 1 \\
\hline Associative Stencil & 1 & 1 \\
\hline Otherwise & $1+2 \times r a d$ & $1+2 \times r a d$ \\
\hline
\end{tabular}


N.5D blocking (mathematical proof provided in [20]) on the same hardware.

Overtile [12], Forma [22] and SDSLc [23] are other frameworks that can accelerate stencil computation by overlapped tiling on GPUs; however, none of them employs dimension streaming. Among non-redundant temporal blocking techniques, trapezoidal tiling [8], diamond tiling [3], and combined diamond and hexagonal tiling [10] have been proposed. Pochoir [32] and YASK [39] are also stencil frameworks that conduct temporal blocking for CPUs and Xeon Phi, respectively. LIFT [11] is a functional data-parallel programming language that allows expressing stencil loops as a set of reusable parallel primitives and optimizing them. Recently, multiple implementations of N.5D blocking on FPGAs have also been proposed with FPGA-specific optimizations [4, 5, 40, 41]. FPGAs tend to achieve better scaling with temporal blocking compared to GPUs due to higher flexibility of employing their on-chip memory which allows larger spatial block sizes compared to GPUs. However, their final performance still falls short of that of modern GPUs due to large gap in peak compute and memory performance.

\section{AN5D Framework}

\subsection{Execution Model}

We denote the temporal blocking size (number of combined iterations/time-steps) as $b_{\mathrm{T}}$, and the spatial blocking (subplane) size as $b_{\mathrm{S}_{i}}$ along each spatial dimension $\left(\mathrm{S}_{i} ; 1 \leq\right.$ $i<N$ ) which excludes the streaming dimension. Since each thread only processes one cell per block, the thread-block size $\left(n_{\mathrm{thr}}\right)$ used by our framework will be $n_{\mathrm{thr}}=\prod_{i=1}^{N-1} b_{\mathrm{S}_{i}}$.

The number of threads per dimension that store updated cells to global memory is represented by $b_{\mathrm{S}_{i}}-2 \times b_{\mathrm{T}} \times r a d$; the difference from $n_{\text {thr }}$ indicates the overlapped (halo) area for each block. We call the non-overlapped region the compute region; these regions cover the entire input grid. The total number of thread-blocks $\left(n_{\mathrm{tb}}\right)$ required for computation is given by $n_{\mathrm{tb}}=\prod_{i=1}^{N-1}\left\lceil\frac{I_{\mathrm{S}_{i}}}{b_{\mathrm{S}_{i}}-2 \times b_{\mathrm{T}} \times r a d}\right]$. Here, we denote the number of iterations for the spatial dimensions (i.e. input grid size) and the time dimension as $I_{S_{i}}$ and $I_{\mathrm{T}}$, respectively.

A sub-plane traverses the streaming dimension from bottom to top, while being accompanied by $b_{\mathrm{T}}$ computational streams, all of which are processed by the same thread-block. Each computational stream undertakes computation of one of the combined time-steps and follows the behavior of the previous time-step's stream. Computing each sub-plane depends on $1+2 \times$ rad sub-planes from the previous time-step. One of these sub-planes is in the same $N$-th spatial position as the current one and rest are the upper and the lower sub-planes. Thus, we store $1+2 \times \mathrm{rad}$ cells in registers for each time-step and for each thread. The streaming latency between time-steps can be seen in Fig. 1. After the first stream $(\mathrm{T}=0)$ reads one sub-plane from global memory, each next time-step updates a successive sub-plane with the distance of rad sub-planes. The size of the region with valid computation, defined as $\prod_{i=1}^{N-1}\left(b_{S_{i}}-2 \times T \times r a d\right)$, gets smaller in accordance with the increased time-step $\left(0<T \leqslant b_{\mathrm{T}}\right)$, and the last computational stream $\left(\mathrm{T}=b_{\mathrm{T}}\right)$ stores the results to global memory only for the non-overlapped area (compute region).

To avoid unnecessary branching and thread-diverges for threads falling inside the halo regions, AN5D overwrites data in these regions with their original values. Furthermore, at the start of the streaming, we use the register variables that are prepared for storing the results of the $\mathrm{T}=b_{\mathrm{T}}-1$ computational stream to maintain the first rad sub-planes on the non-computational space; these sub-planes include the boundary conditions (neighbors for boundary cells). This eliminates the need to reload the same sub-planes from global memory on later time-steps. For the end of the streaming, we instead use the register variables of $\mathrm{T}=0$ to hold these constant boundary sub-planes.

For neighboring accesses on each stencil calculation, threads store data in shared memory as shown in Fig. 3 (a). While updating cells, both register variables and shared memory are accessed. Cell values are fetched from registers if the cells are owned by the requesting threads. Otherwise, the accesses go to shared memory. For diagonal-access free (star) stencils, we eliminate the shared memory use in the upper $\left(\mathrm{S}_{N}=i+1 \sim i+\mathrm{rad}\right)$ and lower sub-planes $\left(\mathrm{S}_{N}=i-\mathrm{rad} \sim i-1\right)$. In the case of associative box stencils, $1+2 \times$ rad consecutive sub-planes are simultaneously updated using values read from one sub-plane. Based on the associativity, each sub-plane is computed through $1+2 \times$ rad partial summations.

(a)

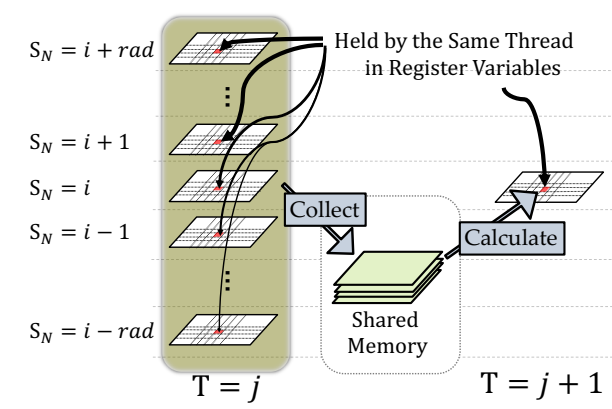

(b)
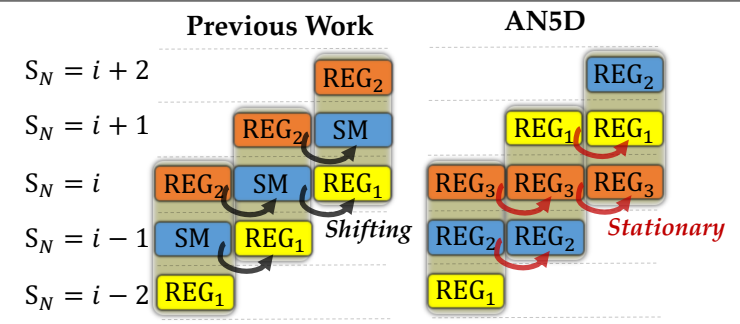

(1) (2)

(3)

(1) (2)

Figure 3. AN5D's on-chip memory management. (a) Shared memory use. (b) Register allocation. 


\subsection{Optimizations}

To enable high degree temporal blocking, AN5D performs several optimizations to expose and efficiently utilize data locality at different levels of the memory hierarchy. To the authors knowledge, this is an unprecedented depth for temporal blocking in GPUs. It is important to note that GPUs are throughput-optimized processors that are commonly regarded as not ideal for the latency-sensitive deep temporal blocking approaches, in comparison to CPUs. The following is list of the key optimizations that allows AN5D to achieve high performance that also scale to double-digit temporal steps in GPUs.

\subsubsection{Register Allocation}

Previous studies of N.5D blocking shift cells through registers (and shared memory) for holding a new sub-plane [20, 24, $26,27]$. AN5D, however, uses a fixed set of registers for each sub-plane value by leveraging the fixed pattern of accesses. Fig. 3 (b) shows the difference between the register allocation of AN5D and previous work. This optimization reduces data stores from $1+2 \times$ rad to 1 for every sub-plane update, leading to less data movement and register usage.

\subsubsection{Double Buffering}

The shared memory use described in this section requires two thread-block synchronizations. One is to wait for the result of the previous time-step, and the other is to avoid overwriting shared memory while other threads are loading from it. The latter synchronization can be skipped by utilizing an additional shared memory buffer to improve performance. Although this increases shared memory requirements, shared memory usage is actually reduced compared to previous work [24, 26] for high degrees of temporal blocking since they use one separate shared memory buffer per combined time-step (see Table 1).

\subsubsection{Division of Streaming Dimension}

Depending on the ratio of input size to block size, there might not be enough thread-blocks participating in the computation to fully utilize the SMs. To improve parallelism, AN5D supports dividing the streaming dimension and processing each stream block using a different thread-block, at the cost of a minor amount of extra redundancy due to overlapping with previous and next stream blocks. This increases the number of thread-blocks by the number of stream blocks as $n_{\mathrm{tb}}^{\prime}=\left\lceil I_{\mathrm{S}_{N}} / h_{\mathrm{S}_{N}}\right\rceil \times n_{\mathrm{tb}}$. Here, $h_{\mathrm{S}_{N}}$ is the

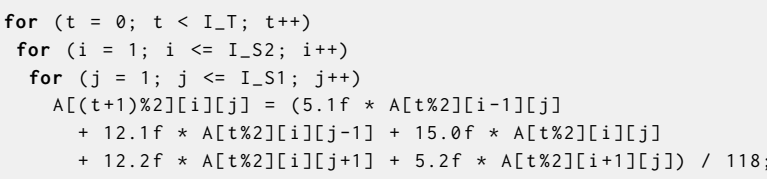

Figure 4. j2d5pt code in C language length of the divided stream blocks. The number of redundant sub-planes between the two consecutive stream blocks is given by $2 \times \sum_{\mathrm{T}=0}^{b_{\mathrm{T}-1}}\left(\mathrm{rad} \times\left(b_{\mathrm{T}}-\mathrm{T}\right)\right)$. The amount of overlapping is variable for different $0 \leqslant T<b_{\mathrm{T}}$, and no overlapping is required for $T=b_{\mathrm{T}}$.

\subsection{Code Generation}

\subsubsection{Host Code}

AN5D generates the host code in the form of repeated kernel calls. Each kernel call performs one temporal blocking solution advancement of size $b_{\mathrm{T}}$. Since our framework requires double-buffered stencil codes that use the modulo operator $(\mathrm{t} \% 2 \rightarrow(\mathrm{t}+1) \% 2,(\mathrm{t}+1) \% 2 \rightarrow \mathrm{t} \% 2)$ as input (example shown in Fig. 4), the final result must be contained in the same global memory space to exactly follow the pattern of the original code. Therefore, we adjust the final block of time-steps by reducing the degree of temporal blocking when $\left(I_{\mathrm{T}} \bmod b_{\mathrm{T}}\right) \neq 0$ or $\left(\left(I_{\mathrm{T}} / b_{\mathrm{T}}\right) \bmod 2\right) \neq\left(b_{\mathrm{T}} \bmod 2\right)$. Since the size of the time dimension is not necessarily known at compile-time, AN5D statically creates the conditional branches.

\subsubsection{Kernel Code}

A kernel generated by AN5D consists of a sequence of macros, each of which computes one sub-plane of a specific time-step. The macro calls take the register variables that point to source and destination sub-planes as input arguments, as well as the streaming index for global memory accesses. Since the generated code performs no data shifting among the variables that are allocated for

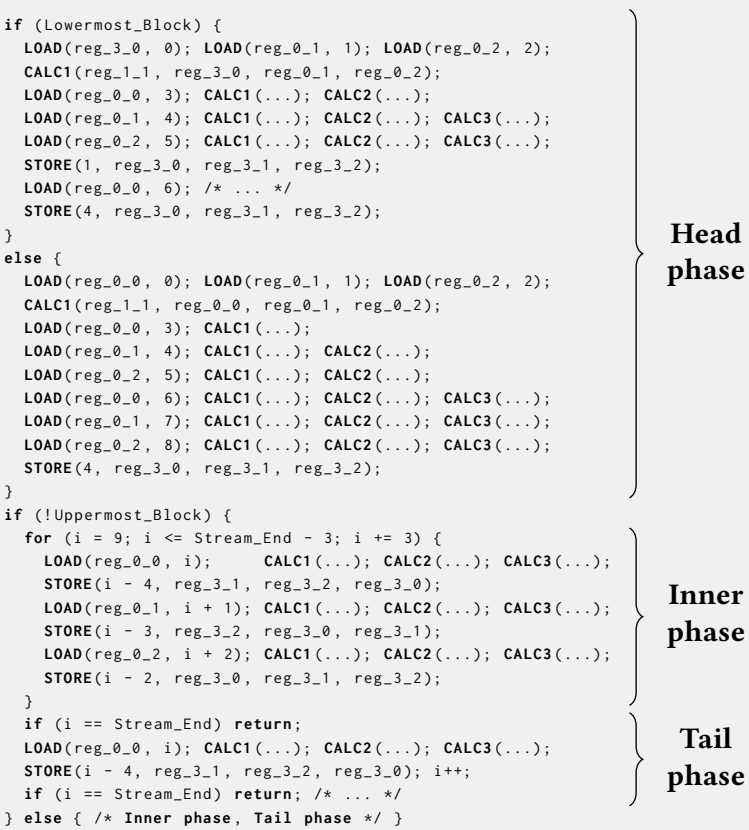

Figure 5. AN5D's generated code $\left(\mathrm{j} 2 \mathrm{~d} 5 \mathrm{pt}, b_{\mathrm{T}}=4\right)$ 
sub-planes, the fixed register allocation depicted in Fig. 3 (b) is encoded as a sequence of macro arguments. The macros generated for each time-step conduct its computation using the same double-buffered shared memory, and the result is written to the destination register variable while avoiding writes to the halo region.

The generated sequence of macros implements the three phases of the computation: head, inner and tail (Fig. 5). As described in Section 4.1, the head phase allocates registers and computes the first rad sub-planes. Since control statements tend to increase register usage, AN5D statically generates the code for this phase instead of using a loop (Lines 2-9 and 12-20 of Fig. 5). For the inner phase, however, since the operations become repetitive, a loop statement is used (Lines 23-30). Here, $N$ and $M$ of reg_N_M represent T of Fig. 1 and the register ID of Fig. 3 (b), respectively. Each macro of LOAD, CALC $(1|2| 3)$ and STORE, loads, computes and stores one sub-plane of the time-step $\mathrm{T}=0 \sim 4$, respectively, while the load, computation and store size is controlled by conditional branches. The macro arguments are sequenced based on the value of $b_{\mathrm{T}}$ and which optimization (associative, diagonal-access free or none) is enabled. Even though this loop statement can be unrolled, our tests showed that doing so results in performance degradation due to increased instruction fetch latency. The tail phase finishes the computation in a similar fashion to the head phase (Lines 31-34).

To reduce register pressure, we disable vectorized shared memory access since extra registers are required to accommodate such accesses. To bypass automated vectorization by NVCC, AN5D performs all shared memory loads through a device function that wraps the shared memory access. In practice, disabling vectorized shared memory accesses not only did not degrade performance, but in fact improved it due to the lowered register pressure allowing higher thread-block-level parallelism. Moreover, AN5D assists constant expansion by the CUDA compiler for double-buffered shared memory accesses by inserting the explicit address or adjusting the number of times the buffer is switched inside the loop statement.

\subsubsection{Implementation}

AN5D generates the necessary CUDA code from an input C description. We use NVIDIA's CUDA compiler for compiling the generated code into an executable. In our implementation, AN5D is integrated into a polyhedral compilation tool called PPCG [35, 36] that supports CUDA code generation. PPCG extracts the polyhedral representation [34] from input (which is currently limited to $C$ language) and this representation mainly consists of three parts which indicate the iteration domain, schedule and array accesses. From these factors, PPCG computes various kinds of dependencies and allows loop rescheduling including general loop tiling and hybrid tiling on the polyhedral representation. Since PPCG's backend is designed for general loop code and can not support the specific forms AN5D requires, we implement a dedicated backend within PPCG for AN5D.

Based on the representation normalized (dead-code eliminated and loop rescheduled) by PPCG's frontend, our backend detects stencil patterns under the following rules:

- The statement describing array accesses is singleton and has only one store access. Moreover, the addresses to read from the array are static.

- All dimensions (time and space) are iterated by one loop, and multi-dimensional array addressing is used rather than linear addressing.

- All the iterations for the spatial dimensions are data independent. Thus, the time loop is the outermost loop. Moreover, the loop after the time loop represents the streaming dimension.

Although our implementation is only tested using inputs that follow the above restrictions, we expect that existing techniques of polyhedral compilation can allow AN5D to accept a wider range of stencil codes.

Parameters such as $b_{\mathrm{T}}, b_{\mathrm{S}_{i}}$ and $h_{\mathrm{S}_{N}}$ are passed as compile-time parameters to our backend, while input size $\left(I_{\mathrm{S}_{i}}\right)$ and time-step count $\left(I_{\mathrm{T}}\right)$ can be modified at run-time. Optimizations such as diagonal-access free and associative stencil optimizations or disabling vectorized shared memory accesses are enabled automatically by our framework but can be disabled using compile-time switches if necessary.

\section{Performance Model}

$b_{\mathrm{S}_{i}}, b_{\mathrm{T}}$ and $h_{\mathrm{S}_{N}}$ need to be tuned in our framework for every given stencil and target hardware to maximize performance. To prune the parameter search space and guide the performance tuning in our framework, we construct a performance model based on the roofline method [37].

First, we calculate the number of threads involved in the computation of any arbitrary stencil supported by our framework, and classify the threads based on the operations that they perform. Operations of importance for us are computation, global memory and shared memory accesses. Based on this, we classify the threads into four categories: out-of-bound, boundary, redundant and valid. Out-of-bound threads are the ones that fall outside of the input grid space due to spatial blocking. These threads still perform writes to shared memory to avoid extra branching in the kernels, but avoid all global memory accesses and computation. Boundary threads are the ones that load the cells holding the input boundary conditions located at the extremes of the input grid. These threads perform shared memory reads and writes and global memory reads but no global memory writes or computation. Redundant and valid threads are also defined as threads that fall inside and outside of halo regions within the spatial blocks, respectively, both of 
which perform shared memory read and write, global memory read, and computation, but only the valid threads perform global memory writes. Based on this classification and considering variable halo size for $\mathrm{T}=0 \sim b_{\mathrm{T}}$ and extra overlapping caused by stream blocking, we develop formulas to calculate the number of threads in each group based on stencil shape, radius and input size, and the aforementioned parameters. We then calculate the total number of threads that perform computation ( $\left.t h_{\text {comp }}\right)$, shared memory read/write $\left(t h_{\text {sm_read }} / t h_{\text {sm_write }}\right)$ and global memory read/write $\left(t h_{\text {gm_read }} / t h_{\text {gm_write }}\right)$.

In the next step, we need to determine how much computation or memory access is performed by each thread. In practice, only one cell is read from global memory per thread when $\mathrm{T}=0$ and one cell is written when $\mathrm{T}=b_{\mathrm{T}}$. Hence, the total global memory traffic (total $\left.{ }_{\mathrm{gm}}\right)$ is $\left(t h_{\text {gm_read }}+t h_{\text {gm_write }}\right) \times n_{\text {word }}$.

For shared memory traffic, we follow a similar approach. Table 2 shows the number of shared memory reads and writes for threads that are involved in such operations. The number of shared memory writes per thread is fixed to one (Section 4.1). For read, the numbers are obtained by deducting the number of accesses that go to registers $(2 \times \mathrm{rad}+1)$ from the total number of cells involved in the stencil computation. In practice, we noticed that the model underestimated performance for box stencils when we used these values. Upon analyzing the generated PTX code we realized that NVCC was automatically caching some of the shared memory data in registers, reducing the number of shared memory reads per thread to one read per column in the stencil. Hence, we divide our expected shared memory reads per thread by $(2 \times \mathrm{rad}+1)$ to get the practical value. Finally, we calculate the total shared memory traffic $\left(\right.$ total $\left._{\mathrm{sm}}\right)$ similar to total $_{\mathrm{gm}}$.

For calculating the total number of floating-point operations, we have to consider the equation associated with each stencil individually. For synthetic star/box stencils in which the computation is a straightforward dot product, all multiplications except the last one are followed by an addition and hence, are merged into FMA operations. However, for stencils such as Jacobi 2D (Fig. 4), since we use --use_fast_math as a compiler switch, we have to consider the alternative implementations of division and sqrt operations enabled by this switch. Specifically, division is implemented as multiplication when this switch is used. Profiling the number of floating-point operations using NVPROF showed that in some cases, for stencils that use an

Table 2. Shared Memory Access per Thread

\begin{tabular}{|c|c|c|c|c|}
\cline { 2 - 5 } \multicolumn{1}{c|}{} & Shape & Read (Expected) & Read (Practical) & Write \\
\hline \multirow{2}{*}{$2 \mathrm{D}$} & Star & $2 \times \mathrm{rad}$ & $2 \times \mathrm{rad}$ & 1 \\
\cline { 2 - 5 } & Box & $(2 \times \mathrm{rad}+1)^{2}-(2 \times \mathrm{rad}+1)$ & $(2 \times \mathrm{rad}+1)-1$ & 1 \\
\hline \multirow{2}{*}{$3 \mathrm{D}$} & Star & $4 \times \mathrm{rad}$ & $4 \times \mathrm{rad}$ & 1 \\
\cline { 2 - 5 } & Box & $(2 \times \mathrm{rad}+1)^{3}-(2 \times \mathrm{rad}+1)$ & $(2 \times \mathrm{rad}+1)^{2}-1$ & 1 \\
\hline
\end{tabular}

equation similar to Jacobi $2 \mathrm{D}$, the compiler expands the statement inside the parenthesis over the division and when the division is replaced with multiplication, the multiplications and additions are merged into FMA operations. Based on these transformations, we determine the mapping of each stencil's equation to ADD, MUL and FMA operations and knowing the number of threads involved in the computation, we calculate the total number of floating-point operations that are performed (total $l_{\text {comp }}$ ).

In the next step, we consider three possible bottleneck points: compute, global memory, and shared memory. We ignore registers as a bottleneck since we assume that, as long as no register spilling occurs, the register bandwidth is high enough not to become a bottleneck. We calculate the expected kernel run time for each level of bottleneck by dividing the total traffic/computation involved for that level by its associated peak performance. For compute, we use the theoretical peak compute performance of the devices (peak $\left.k_{\text {comp }}\right)$. However, this peak performance is only valid if all the computation is mapped to FMA operations. Hence, we calculate ALU utilization efficiency as $e f f_{\mathrm{ALU}}=\frac{2 \times o p_{\mathrm{FMA}}+o p_{\mathrm{MUL}}+o p_{\mathrm{ADD}}+o p_{\mathrm{OTHER}}}{2 \times\left(o p_{\mathrm{FMA}}+o p_{\mathrm{MUL}}+o p_{\mathrm{ADD}}+o p_{\mathrm{OTHER}}\right)}$.

Then, we calculate run time assuming compute-bound operation as time $_{\text {comp }}=\frac{\text { total }_{\text {comp }}}{\text { peak } k_{\mathrm{comp}} \times e f f_{\mathrm{ALU}}}$. For shared and global memory, we use open-source benchmarks to measure practical peak performance $\left(p e a k_{\mathrm{sm} \mid \mathrm{gm}}\right)$ on the GPUs; specifically, gpumembench [15] for measuring shared memory bandwidth (after adjusting the default vector size), and BabelStream [6] for measuring global memory bandwidth. Since the measured peak performance for both memory types was different depending on data type (with the difference being relatively large for shared memory), we used the associated peak values depending on the data type used in the computation. Run times assuming global and shared memory bottleneck $\left(\right.$ time $\left._{\mathrm{gm} \mid \mathrm{sm}}\right)$ were then calculated by dividing total $_{\mathrm{sm} \mid \mathrm{gm}}$ by peak $k_{\mathrm{sm} \mid \mathrm{gm}}$.

One more point needs to be considered before we can calculate the expected run time: SM utilization efficiency $\left(e f f_{\mathrm{SM}}\right)$. This value depends on how many thread-blocks are involved in the computation $\left(n_{\mathrm{tb}}^{\prime}\right)$ and how many SMs exist on the device $\left(n_{\mathrm{SM}}\right)$. The number of thread-blocks that can simultaneously reside on each SM is limited by two factors: the hardware limit of 2048 threads per SM, and the 64|96 KB shared memory size per SM on modern NVIDIA GPUs. The former factor limits the number of concurrent threadblocks per SM to $2048 / n_{\text {thr }}$ while for diagonally-access free and associative stencils, the latter factor imposes a limit of $\frac{64 \mathrm{~KB} \text { (or } 96 \mathrm{~KB})}{2 \times n_{\text {thr }} \times n_{\text {word }}}$ thread-blocks (See Table 1 ). In practice, even for double-precision data, the former limit will be smaller and hence, we calculate SM utilization efficiency as $e f f_{\mathrm{SM}}=$ $\left\lfloor\frac{n_{\mathrm{tb}}^{\prime}}{2048 / n_{\mathrm{thr}}} \mid /\left[\frac{n_{\mathrm{tb}}^{\prime}}{2048 / n_{\mathrm{thr}}}\right\rceil\right.$. Finally, we calculate expected run time as

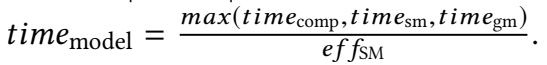




\section{Methodology}

\subsection{Benchmarks}

We evaluate our framework using a wide range of synthetic and general stencil benchmarks shown in Table 3. In this table, $c$ denotes compile-time constant coefficients and $x$ denotes the radius for the synthetic stencils. All other stencils have a radius of one except j2d9pt which is a $2^{\text {nd }}$-order stencil. The synthetic benchmarks include all single-array single-statement box and star stencil shapes from 1st to 4th-order. These benchmarks are specifically chosen to allow fair comparison with previous work; some have a same-shaped equivalent among the synthetic benchmarks but different computation pattern.

For each benchmark, we wrote $\mathrm{C}$ code and then generate the associated CUDA host and kernel code for AN5D, general loop tiling, and hybrid tiling from the same input code. To enable loop unrolling optimization for loop tiling and hybrid tiling, we have to use static input sizes in the source code; this means that the source code needs to be recompiled each time the input size needs to be changed which limits the usability of these methods for real-world applications. However, our framework does not suffer from this limitation. For STENCILGEN, we use the kernels released by the authors, available at https://github.com/ pssrawat/IEEE2017. Comparison between frameworks is limited to stencil types available in this repository.

We use an input size of $16,384^{2}$ for $2 \mathrm{D}$ and $512^{3}$ for $3 \mathrm{D}$ stencils with 1,000 iterations. These input sizes align with previous work [24, 25] and are specifically chosen so that the GPUs are well-utilized and measured performance is stable, without unnecessarily increasing the benchmark time. We also evaluate all benchmarks with both single-precision and double-precision floating-point cell values. Each benchmark is repeated five times (on top of an initial warm-up run) and the average performance is reported (excluding PCI-E transfer time). Minimum run time for one instance of each benchmark is $400 \mathrm{~ms}$.

\subsection{Hardware and Software}

We evaluate all benchmarks on the latest NVIDIA Tesla architectures: Pascal and Volta . The specifications of these cards are shown in Table 4. As for the external memory and shared memory bandwidth of the GPUs, we use open-source benchmarks to measure practical peak performance on the GPUs; specifically, gpumembench [15] for measuring shared memory bandwidth (after adjusting the default vector size), and BabelStream [6] for measuring global memory bandwidth.

Both of our machines use CentOS 7.6 and Intel Xeon processors. For compiling the kernel codes, we use CUDA 10.0.130 and associated NVIDIA driver v410.48. We compile all kernels using the following set of compiler arguments: "-gencode=arch=compute_(60|70), code=sm_(60|70) -use_fast_math -Xcompiler -03 -fopenmp". Even though -use_fast_math can reduce the numerical accuracy of the results, we use this switch so that our evaluation aligns with previous work [24, 25]. Moreover, for cases where complex mathematical operations are involved (sqrt and division), not using this switch might make the benchmark compute-bound and eliminate the need for shared memory and register-based optimizations.

\subsection{Parameter Tuning}

We measure the performance of AN5D using two configurations. The first configuration called Sconf uses the same kernel parameters as STENCILGEN: $b_{\mathrm{T}}=4, h_{\mathrm{S}_{N}}=128$ and $b_{S_{i}}=\{32 \mid 128\}(2 \mathrm{D} \mid 3 \mathrm{D}$, respectively). We also disable associative stencil optimization for $2 \mathrm{D}$ stencils and streaming division for 3D stencils in this configuration since STENCILGEN does not use this optimization for such stencils. The second configuration is called Tuned. For this configuration, we first use our model to predict the performance for all valid parameter sets. Specifically, we use $b_{\mathrm{T}} \in[1,16]$ for $2 \mathrm{D}$, and $b_{\mathrm{T}} \in[1,8]$ for $3 \mathrm{D}$ stencils, respectively. $b_{\mathrm{S}_{i}}$ for $2 \mathrm{D}$ stencils is chosen from the set of $\{128,256,512\}$, and for $3 \mathrm{D}$, is chosen from $\{16 \times 16,32 \times 16$, $32 \times 32,64 \times 16\} . h_{S_{N}}$ is also chosen from the sets of $\{256,512,1024\}$ and $\{128,256\}$ for $2 \mathrm{D}$ and $3 \mathrm{D}$ stencils, respectively. These settings result in 144 configurations for $2 \mathrm{D}$, and 64 configurations for 3D stencils per GPU, all of which can be searched in a few seconds using our model. However, we experimentally find that a minimum of

Table 3. Benchmarks

\begin{tabular}{|c|c|c|}
\hline Stencil & Computation & FLOP/Cell \\
\hline $\begin{array}{l}\operatorname{star} 2 d\{x\} r \\
x \in[1,4]\end{array}$ & $\begin{array}{l}c_{(x, y)} f_{(x, y)}+\sum_{i=-x, i \neq 0}^{x} \\
\left(c_{(x+i, y)} f_{(x+i, y)}+c_{(x, y+i)} f_{(x, y+i)}\right)\end{array}$ & $8 x+1$ \\
\hline $\begin{array}{l}\operatorname{box} 2 d\{x\} r \\
x \in[1,4]\end{array}$ & $\sum_{i=-x}^{x} \sum_{j=-x}^{x} c_{(x+i, y+j)} f_{(x+i, y+j)}$ & $\begin{array}{c}2 \times \\
(2 x+1)^{2}-1\end{array}$ \\
\hline $\mathrm{j} 2 \mathrm{~d} 5 \mathrm{pt}$ & $\begin{array}{l}\left(c_{(x, y)} f_{(x, y)}+\sum_{i=-1, i \neq 0}^{1}\right. \\
\left.\left(c_{(x+i, y)} f_{(x+i, y)}+c_{(x, y+i)} f_{(x, y+i)}\right)\right) / c_{0}\end{array}$ & 10 \\
\hline j2d9pt & $\begin{array}{l}\left(c_{(x, y)} f_{(x, y)}+\sum_{i=-2, i \neq 0}^{2}\right. \\
\left.\left(c_{(x+i, y)} f_{(x+i, y)}+c_{(x, y+i)} f_{(x, y+i)}\right)\right) / c_{0}\end{array}$ & 18 \\
\hline j2d9pt-gol & $\left(\sum_{i=-1}^{1} \sum_{j=-1}^{1} c_{(x+i, y+j)} f_{(x+i, y+j)}\right) / c_{0}$ & 18 \\
\hline gradient $2 \mathrm{~d}$ & $\begin{array}{l}c_{(x, y)} f_{(x, y)}+1.0 / \operatorname{sqrt}\left(c_{0}+\sum_{i=-1, i \neq 0}^{1}\right. \\
\left(\left(f_{(x, y)}-f_{(x+i, y)}\right) \times\left(f_{(x, y)}-f_{(x+i, y)}\right)+\right. \\
\left.\left.\left(f_{(x, y)}-f_{(x, y+i)}\right) \times\left(f_{(x, y)}-f_{(x, y+i)}\right)\right)\right)\end{array}$ & 19 \\
\hline $\begin{array}{l}\operatorname{star} 3 d\{x\} r \\
x \in[1,4]\end{array}$ & $\begin{array}{l}c_{(x, y)} f_{(x, y)}+\sum_{i=-x, i \neq 0}^{x}\left(c_{(x+i, y, z)} f_{(x+i, y, z)}+\right. \\
\left.c_{(x, y+i, z)} f_{(x, y+i, z)}+c_{(x, y, z+i)} f_{(x, y, z+i)}\right)\end{array}$ & $12 x+1$ \\
\hline $\begin{array}{l}\operatorname{box} 3 d\{x\} r \\
x \in[1,4]\end{array}$ & $\sum_{i=-x}^{X} \sum_{j=-x}^{X} \sum_{k=-x}^{X} c_{(x+i, y+j, z+k)} f_{(x+i, y+j, z+k)}$ & $\begin{array}{c}2 \times \\
(2 x+1)^{3}-1\end{array}$ \\
\hline $\mathrm{j} 3 \mathrm{~d} 27 \mathrm{pt}$ & $\begin{array}{l}\left(\sum_{i=-1}^{1} \sum_{j=-1}^{1} \sum_{k=-1}^{1} c_{(x+i, y+j, z+k)}\right. \\
\left.f_{(x+i, y+j, z+k)}\right) / c_{0}\end{array}$ & 54 \\
\hline
\end{tabular}


Table 4. GPU Specifications (Float | Double)

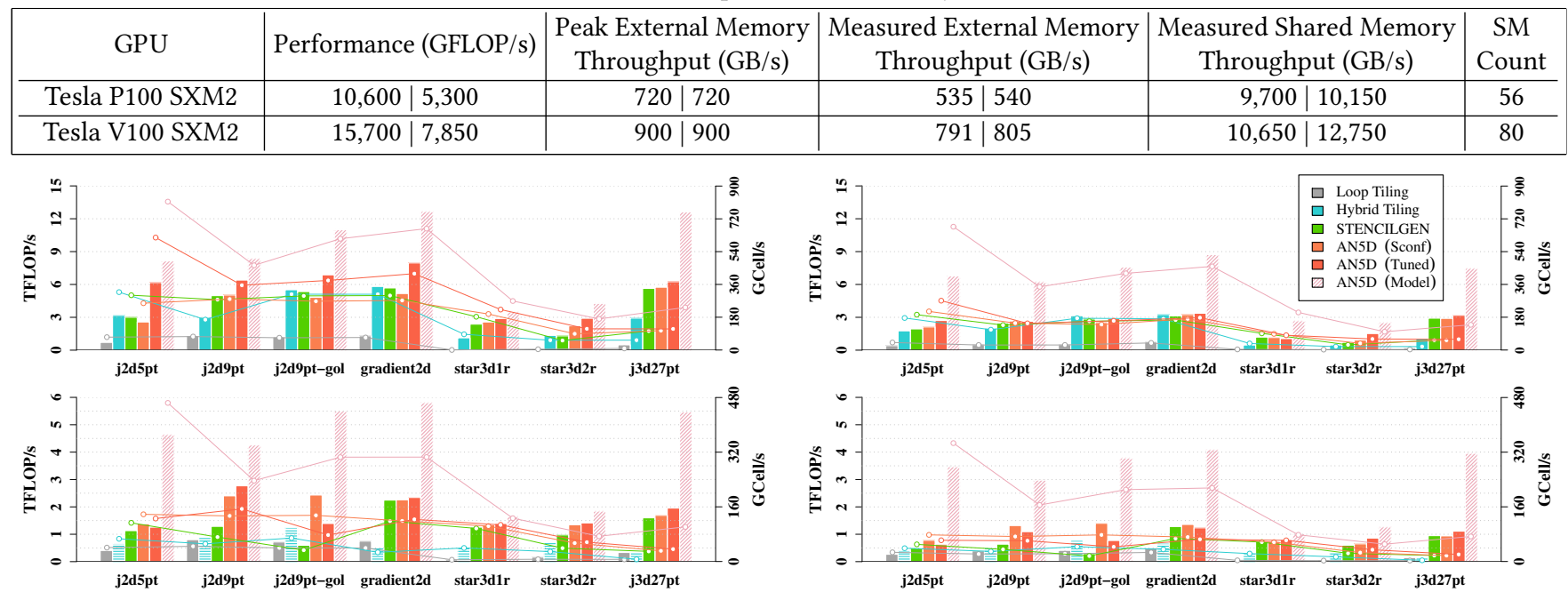

Figure 6. Performance on Tesla V100 (left) and P100 (right) with float (top) and double (bottom) data types

$b_{\mathrm{T}} \times(2 \times r a d+1)+b_{\mathrm{T}}+20$ and $2 \times b_{\mathrm{T}} \times(2 \times r a d+1)+b_{\mathrm{T}}+30$ registers are used per thread, for single and double-precision data types, respectively. Hence, we use these limits to prune configurations which are expected to require more than the hardware limits of 255 registers per thread or 65,536 registers per SM. Then, we sort the parameter sets based on the performance predicted by our model and choose the top 5 for each GPU. Finally, we run these configurations on the GPUs and choose the one that achieves the highest measured performance in each case.

For loop tiling, we use the default tiling size that is provided by PPCG. Hybrid tiling provides parameters to optimize tile sizes along each time/spatial dimension, and thread-block sizes along each spatial dimension. We conduct a large-scale parameter search to find the optimal parameters for each combination of stencil pattern and GPU. Here, around 10,000 and 5,000 parameter configurations are explored for each $2 \mathrm{D}\left(b_{\mathrm{T}}=[2,20], b_{\mathrm{S}}=[1,32] \times[32,2048]\right.$, $\left.n_{\mathrm{thr}}=[1,32] \times[32,1024]\right)$ and $3 \mathrm{D}$ stencil $([2,12]$, $[1,4] \times[1,32] \times[32,256],[1,4] \times[1,32] \times[32,256])$, respectively. We set $8,192^{2}$ and $512^{3}$ as $2 \mathrm{D} / 3 \mathrm{D}$ grid size and 120 as iteration count for parameter search. We conduct the parameter search with single-precision and reuse the optimal parameters of each stencil for double-precision. Even though this might not necessarily result in the best performance for the double-precision case, the method would be the same as STENCILGEN and the Sconf configuration for AN5D where the same configurations are used for single and double-precision data types. To speed-up parameter tuning for the Tesla V100 GPU, we utilize the ABCI supercomputer environment with CUDA 9.2, while parameter tuning for Tesla P100 and the actual performance measurement for both GPUs are done using the same local machine as we use for other frameworks with CUDA 10.0.130.
One final parameter to tune is the number of registers allocated per thread. This value can be restricted using the NVCC option -maxrregcount. Limiting register usage can allow more thread-blocks to reside on the same SM at the same time, leading to higher parallelism and performance. However, this restriction can also lead to register spilling which would adversely affect performance. We encountered multiple cases where limiting register usage per thread reduced register usage without spilling and consequently, increased performance due to better SM utilization. Hence, for all benchmarks and all frameworks, apart from the standard compilation where no register limit was imposed, we also generated binaries with limits of 32 and 64 registers per thread, and chose the best performance for each case. For the Tuned configuration of AN5D, we further added the limit of 96 register per thread since it proved to be useful for high-order stencils and high-degree temporal blocking.

\section{Results}

\subsection{Comparison}

Fig. 6 shows performance comparison results for multiple stencils. Model is the performance predicted by our model for the Tuned configuration. On Tesla V100, taking both Sconf and Tuned results into account, AN5D achieves the highest performance for both single and double-precision. On Tesla P100, AN5D achieves the highest performance except in the cases of $\mathrm{j} 2 \mathrm{~d} 9 \mathrm{pt}$-gol and star3d1r in single-precision where hybrid tiling and STENCILGEN are faster than AN5D by $8 \%$ and $3 \%$, respectively. These two cases will be further discussed later in this section.

Using the same configuration as STENCILGEN, AN5D improves performance for most cases and specifically exhibits large performance improvements of up to $2 \mathrm{x}$ for double-precision benchmarks due to lower register pressure than STENCILGEN. This shows that even though the focus 
Table 5. AN5D Configuration and Performance (Regs: Optimal register limitation per thread, “-”: no limitation, Tuned \& Model: GFLOP/s)

\begin{tabular}{|c|c|c|c|c|c|c|c|c|c|c|c|c|c|c|c|c|c|c|c|c|c|c|c|c|}
\hline \multirow{2}{*}{ Pattern } & \multicolumn{6}{|c|}{ Tesla V100 (float) } & \multicolumn{6}{|c|}{ Tesla V100 (double) } & \multicolumn{6}{|c|}{ Tesla P100 (float) } & \multicolumn{6}{|c|}{ Tesla P100 (double) } \\
\hline & $b_{\mathrm{T}}$ & $b_{\mathrm{S}}$ & $h_{\mathrm{S}_{N}}$ & Regs & Tuned & Model & $b_{\mathrm{T}}$ & $b_{\mathrm{S}}$ & $h_{\mathrm{S}_{N}}$ & Regs & Tuned & Model & $b_{\mathrm{T}}$ & $b_{\mathrm{S}}$ & $h_{\mathrm{S}_{N}}$ & Regs & Tuned & Model & $b_{\mathrm{T}}$ & $b_{\mathrm{S}}$ & $h_{\mathrm{S}_{N}}$ & Regs & Tuned & Model \\
\hline star2d1r & 10 & 256 & 256 & 64 & 5,631 & 7,330 & 10 & 256 & 256 & - & 3,306 & 4,177 & 13 & 512 & 256 & - & 2,507 & 6,091 & 15 & 128 & 1024 & - & 1,588 & 3,118 \\
\hline star2d2r & 10 & 512 & 256 & 64 & 6,319 & 8,172 & 6 & 512 & 128 & 64 & 3,071 & 4,431 & 10 & 512 & 512 & - & 2,576 & 7,698 & 8 & 512 & 512 & 96 & 1,397 & 4,042 \\
\hline $\operatorname{star} 2 \mathrm{~d} 3 \mathrm{r}$ & 7 & 512 & 256 & 64 & 7,132 & 8,627 & 6 & 256 & 128 & 96 & 3,221 & 4,707 & 6 & 512 & 512 & 96 & 3,424 & 8,144 & 7 & 256 & 256 & - & 1,912 & 3,857 \\
\hline star2d4r & 5 & 512 & 256 & - & 7,244 & 8,954 & 4 & 256 & 128 & 96 & 3,422 & 4,680 & 5 & 512 & 512 & - & 3,573 & 8,405 & 5 & 512 & 512 & - & 1,956 & 4,397 \\
\hline $\mathrm{bo} \times 2 \mathrm{~d} 1 \mathrm{r}$ & 10 & 256 & 256 & 96 & 6,693 & 11,327 & 10 & 256 & $256^{\circ}$ & 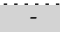 & 2,984 & 5,664 & 10 & 256 & 512 & 64 & 2,823 & 7,804 & $\ddot{8}$ & 128 & 128 & 96 & 1,959 & 3,660 \\
\hline bo $\times 2 \mathrm{~d} 2 \mathrm{r}$ & 5 & 256 & 256 & 64 & 9,163 & 12,473 & 3 & 256 & 128 & 64 & 4,686 & 5,858 & 5 & 256 & 512 & 64 & 4,626 & 8,578 & 5 & 256 & 512 & - & 2,673 & 4,289 \\
\hline bo $\times 2 \mathrm{~d} 3 \mathrm{r}$ & 2 & 256 & 128 & 96 & 10,227 & 12,391 & 2 & 256 & 128 & 64 & 5,507 & 6,196 & 2 & 256 & 128 & 96 & 5,598 & 8,584 & 2 & 128 & 128 & 96 & 3,652 & 4,244 \\
\hline bo $\times 2 d 4 r$ & 4 & 512 & 256 & 96 & 10,772 & 13,241 & 1 & 256 & 128 & 96 & 5,770 & 6,576 & 4 & 512 & 512 & 96 & 6,546 & 9,174 & 1 & 128 & 128 & 96 & 3,921 & 4,556 \\
\hline $\mathrm{j} 2 \mathrm{~d} 5 \mathrm{pt}$ & 10 & 256 & 256 & 64 & 6,160 & 8,144 & 10 & 256 & 256 & 96 & 1,258 & 4,642 & 13 & 512 & 256 & - & 2,708 & 6,768 & 15 & 128 & 1024 & - & 621 & 3,465 \\
\hline j2d9pt & 5 & 256 & 256 & - & 6,398 & 8,370 & 5 & 256 & 256 & 64 & 2,770 & 4,259 & 10 & 512 & 256 & 64 & 2,635 & 6,244 & 6 & 512 & 128 & 64 & 1,093 & 2,976 \\
\hline j2d9pt-gol & 10 & 256 & 256 & - & 6,865 & 10,994 & 10 & 256 & 256 & - & 1,394 & 5,497 & 10 & 256 & 512 & 64 & 2,883 & 7,575 & 10 & 256 & 512 & - & 770 & 3,787 \\
\hline gradient $2 \mathrm{~d}$ & 10 & 256 & 256 & 96 & 7,965 & 12,660 & 8 & 256 & 128 & 64 & 2,343 & 5,806 & 10 & 256 & 512 & 64 & 3,369 & 8,723 & 8 & 128 & 128 & 96 & 1,234 & 4,091 \\
\hline star $3 \mathrm{~d} 1 \mathrm{r}$ & 4 & $32 \times 32$ & 128 & 96 & 2,887 & 3,498 & 4 & $64 \times 16$ & 128 & 32 & 1,393 & 1,647 & 5 & $32 \times 32$ & 128 & 96 & 1,055 & 2,682 & 3 & $32 \times 32$ & 128 & 32 & 805 & 1,015 \\
\hline star3d2r & 3 & $32 \times 32$ & 128 & 32 & 2,910 & 4,268 & 2 & $32 \times 32$ & 128 & 64 & 1,413 & 1,847 & 2 & $32 \times 32$ & 128 & 96 & 1,545 & 2,512 & 2 & $32 \times 32$ & 128 & 32 & 859 & 1,268 \\
\hline star3d3r & 2 & $32 \times 32$ & 128 & 32 & 3,118 & 4,518 & 2 & $32 \times 32$ & 256 & 96 & 1,591 & 2,193 & 2 & $32 \times 32$ & 128 & 32 & 1,523 & 3,117 & 2 & $32 \times 32$ & 256 & - & 966 & 1,528 \\
\hline star3d4r & 2 & $32 \times 32$ & 256 & 32 & 2,808 & 4,063 & 1 & $32 \times 32$ & 128 & 96 & 1,684 & 2,087 & 1 & $64 \times 16$ & 128 & - & 1,656 & 2,824 & 1 & $32 \times 32$ & 256 & 64 & 1,135 & 1,354 \\
\hline $\mathrm{bo} \times 3 \mathrm{~d} 1 \mathrm{r}$ & 3 & $32 \times 32$ & 256 & 32 & 6,284 & 12,811 & 3 & $32 \times 16$ & 128 & 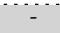 & 2,888 & 5,552 & 3 & $32 \times 32$ & 256 & 64 & 3,168 & 7,590 & 3 & $32 \times 32$ & 128 & 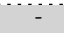 & 1,671 & 4,015 \\
\hline bo $\times 3 \mathrm{~d} 2 \mathrm{r}$ & 1 & $32 \times 16$ & 128 & 96 & 8,666 & 13,640 & 1 & $32 \times 16$ & 128 & 96 & 5,024 & 6,820 & 1 & $32 \times 16$ & 128 & 64 & 5,528 & 9,482 & 1 & $32 \times 16$ & 128 & 96 & 3,189 & 4,741 \\
\hline bo $\times 3 d 3 r$ & 1 & $64 \times 16$ & 128 & 96 & 9,351 & 13,931 & 1 & $32 \times 16$ & 128 & - & 2,993 & 7,599 & 1 & $32 \times 16$ & 128 & 96 & 6,401 & 9,749 & 1 & $32 \times 16$ & 128 & - & 1,934 & 4,874 \\
\hline bo $\times 3 \mathrm{~d} 4 \mathrm{r}$ & 1 & $64 \times 16$ & 256 & - & 9,707 & 15,248 & 1 & $64 \times 16$ & 256 & - & 4,635 & 7,624 & 1 & $32 \times 16$ & 128 & - & 3,056 & 9,928 & 1 & $16 \times 16$ & 256 & - & 794 & 4,891 \\
\hline $\mathrm{j} 3 \mathrm{~d} 27 \mathrm{pt}$ & 3 & $32 \times 32$ & 256 & 32 & 6,251 & 12,617 & 3 & $32 \times 16$ & 128 & 64 & 1,957 & 5,468 & 3 & $32 \times 32$ & 256 & 96 & 3,183 & 7,476 & 3 & $32 \times 32$ & 128 & 64 & 1,112 & 3,954 \\
\hline
\end{tabular}

of our framework is to enable high-degree temporal blocking, it can still compete with or outperform state-of-the-art using same configurations. Fig. 7 shows the register usage of STENCILGEN and Sconf configuration for AN5D for multiple stencils. Even though in theory, AN5D requires $b_{\mathrm{T}}$ extra registers per thread for sub-plane management compared to STENCILGEN, in practice it uses fewer registers on average. Moreover, when we limit register usage per thread to 32 (maximum value to achieve $100 \%$ SM occupancy), none of the seven binaries generated by AN5D cause register spilling, while STENCILGEN causes spilling for the second-order stencils ( $\mathrm{j} 2 \mathrm{~d} 9 \mathrm{pt}$ and star3d2r).

Loop tiling fails to compete with any of the evaluated frameworks. Hybrid tiling, on the other hand, achieves competitive performance for $2 \mathrm{D}$ stencils but only outperforms AN5D in the case of j2d9pt-gol on P100. Based on profiling results, hybrid blocking achieves better global memory performance than AN5D in this case, leading to slightly higher performance. For 3D stencils, this tiling method falls short of STENCILGEN and AN5D since, as discussed in Section 3, it is limited to smaller block sizes due to lack of dimension streaming.

Comparing the Tuned and Sconf configurations for AN5D, the Tuned configuration increases the performance in every case for single-precision benchmarks on Tesla V100. For double-precision, however, performance degradation is observed for multiple stencils on both devices. Further analysis showed that the CUDA compiler

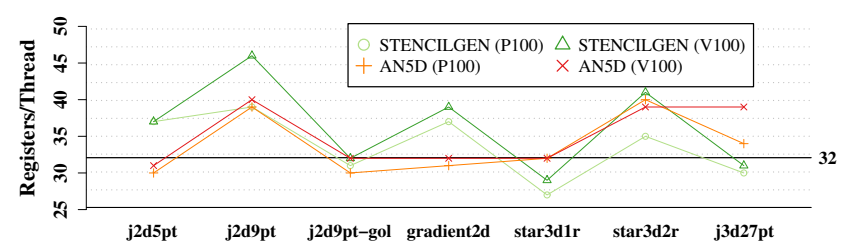

Figure 7. Register usage with no register limitation (float) generates inefficient machine code for stencils which use double-precision division ( $\mathrm{j} * * * *$ stencils in Fig. 6 ), resulting in noticeable slow-down compared to same-shaped stencils that do not use this operation (Fig. 9). Since our model is unaware of this problem, it fails to predict the best configuration for such stencils. We experimentally discovered that replacing the double-precision division " $/ N$ " with " $\times(1 / N)$ " can be used as a work-around here, but we did not use it in our evaluation to keep the comparison fair.

\subsection{Model Accuracy}

Table 5 shows the best-performing AN5D configuration among top 5 configurations predicted by our model for all our evaluated stencils, alongside with optimal register per thread limit (Regs), and measured (Tuned) and predicted (Model) performance for this configuration. We define model accuracy as the ratio of Tuned to Model. Our model exhibits an average accuracy of $49 \%(16 \sim 86 \%)$ on P100 and $67 \%(25 \sim 89 \%)$ on V100 when all cases from Table 5 are considered. Excluding the benchmarks that use the division operation and achieve lower-than-expected performance with double-precision data, the model accuracy improves to $53 \%(16 \sim 86 \%)$ and $71 \%(39 \sim 89 \%)$, on P100 and V100, respectively. The lowest accuracy values are obtained for box3d3r and box3d4r where register usage that is not considered in our model becomes a bottleneck, especially on the P100 device. Since our model predicts shared memory as the performance bottleneck in every case except box $3 \mathrm{~d} 3 \mathrm{r}$ and box $3 \mathrm{~d} 4 \mathrm{r}$, the model accuracy can be considered as an estimation for shared memory efficiency on these GPUs. Profiling multiple of our benchmarks on P100 with the same kernel that was used on V100 showed that P100 achieves less than half the shared memory bandwidth of V100 for the same kernels, despite the fact that the difference between the measured shared memory bandwidth of these devices is less than $10 \%$ (Table 4). Hence, we can conclude that Tesla 
V100 is more suitable for N.5D blocking as it achieves higher shared memory efficiency, pointing to a more efficient shared memory architecture and controller on this device compared to Tesla P100. One negative side-effect of the lower-than-expected shared memory efficiency of P100 is that our model tends to overestimate the optimal degree of temporal blocking on this device. For example, for the star3d1r benchmark on P100 which is the only case we report lower performance than STENCILGEN, if we use the exact same configuration as reported in Table 5 but reduce $b_{\mathrm{T}}$ to 3 , performance will increase to $1,263 \mathrm{GFLOP} / \mathrm{s}$ which is higher than STENCILGEN, enabling us to achieve higher performance than this state-of-the-art framework for every benchmark and device. It is likely that if we consider the shared memory efficiency of the devices in our model, we can find better configurations also for other benchmarks.

\subsection{Scaling Performance}

Fig. 8 shows performance scaling with $b_{\mathrm{T}}$ on Tesla V100 when we fix the Tuned parameters except register limitation which is tuned for each $b_{\mathrm{T}}$. As our model predicted, performance of $2 \mathrm{D}$ stencils scales up to $b_{\mathrm{T}}=10,3 \mathrm{D}$ star stencils up to $b_{\mathrm{T}}=5$, and $3 \mathrm{D}$ box stencils up to $b_{\mathrm{T}}=3$. This shows that our framework is successful in optimizing different stencil types for high-degree temporal blocking, and that our model is successful in predicting the trend of performance variations.

Fig. 9 provides the scaling performance from first-order to fourth-order stencils on Tesla V100. The best performance of first-order stencils is gained with high-degree temporal blocking sizes (2D: 8 15, 3D: 3 5). For 2D stencils and 3D star stencils, most cases including fourth-order stencils achieved the best performance with
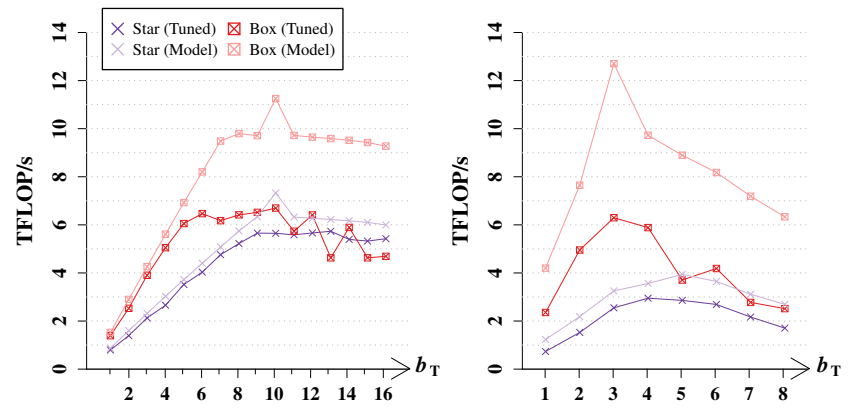

Figure 8. Scaling with Degree of Temporal Blocking on Tesla V100 with 2D (left) and 3D (right) stencils. Float, $\mathrm{rad}=1$.

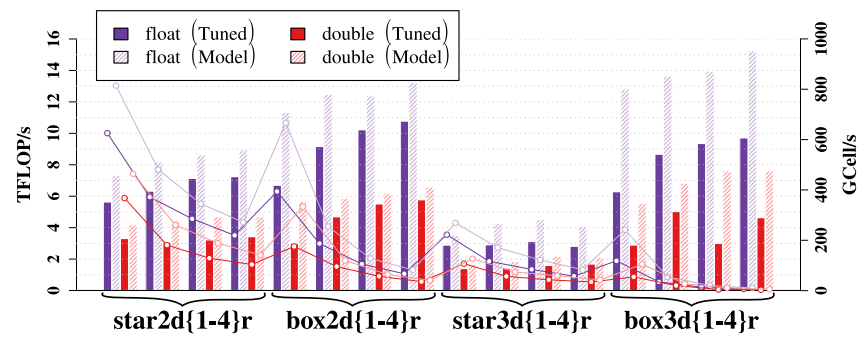

Figure 9. Performance of Star/Box Stencils on Tesla V100
$b_{\mathrm{T}}>=2$. The only exception is high-order 3D box stencils where register pressure and the ratio of halo size to spatial block size is too high to allow performance scaling with temporal blocking. Though for these stencils we still achieved around $60 \%$ of the peak compute performance on Tesla V100 without temporal blocking. It is noteworthy that our framework is the only framework that has achieved high-performance high-order stencil computation with multi-degree temporal blocking so far. Rawat et al. [25] recently proposed a reordering framework to specifically target high-order stencils that benefit less from temporal blocking. We compiled and executed the most compute-intensive 3D single-array benchmark (3d125pt) from their public repository (https://github.com/pssrawat /ppopp-artifact) which achieved $41 \%$ of the peak (double-precision) compute performance on Tesla V100, while AN5D achieved $51 \%$ of the peak without temporal blocking. This indicates that even for high-order stencils where temporal blocking is not necessarily applicable, our framework can achieve higher computational efficiency compared to state-of-the-art.

\section{Conclusion}

In this paper we presented AN5D, our stencil framework for high-degree temporal blocking on GPUs. With careful register-level optimizations and shared memory double-buffering, we managed to implement temporal blocking as a practical optimization for low and mid-order stencils, while improving computational efficiency for high-order stencils where temporal blocking is less applicable. Moreover, our performance model allowed us to quickly choose the best configuration for each stencil pattern and facilitate portable high performance among different GPUs. We showed the efficiency of our framework with respect to register pressure and performance scaling with high degrees of temporal blocking, and, through a comprehensive comparison with previous work using typical stencil patterns, we demonstrated that temporal blocking is crucial to achieve high computational performance for stencil computation.

For future work, we plan to add support for source code transformation techniques such as warp specialization and idle-wrap elimination to our framework to potentially enable lower register pressure and better shared memory efficiency, and implement multi-output temporal blocking to optimize multi-statement stencils.

\section{Acknowledgement}

This work was partially supported by JST-CREST under Grant Number JPMJCR19F5. Computational resource of AI Bridging Cloud Infrastructure (ABCI) provided by National Institute of Advanced Industrial Science and Technology (AIST) was used. 


\section{References}

[1] Y. Ao, C. Yang, X. Wang, W. Xue, H. Fu, F. Liu, L. Gan, P. Xu, and W. Ma. 2017. 26 PFLOPS Stencil Computations for Atmospheric Modeling on Sunway TaihuLight. In 2017 IEEE International Parallel and Distributed Processing Symposium (IPDPS). 535-544. https://doi. org/10.1109/IPDPS.2017.9

[2] Uday Bondhugula, Vinayaka Bandishti, and Irshad Pananilath. 2017. Diamond Tiling: Tiling Techniques to Maximize Parallelism for Stencil Computations. IEEE Trans. Parallel Distrib. Syst. 28, 5 (May 2017), 1285-1298. https://doi.org/10.1109/TPDS.2016.2615094

[3] U. Bondhugula, V. Bandishti, and I. Pananilath. 2017. Diamond Tiling: Tiling Techniques to Maximize Parallelism for Stencil Computations. IEEE Transactions on Parallel and Distributed Systems 28, 5 (May 2017), 1285-1298. https://doi.org/10.1109/TPDS.2016.2615094

[4] Y. Chi, J. Cong, P. Wei, and P. Zhou. 2018. SODA: Stencil with Optimized Dataflow Architecture. In 2018 IEEE/ACM International Conference on Computer-Aided Design (ICCAD). 1-8. https://doi.org/ 10.1145/3240765.3240850

[5] Johannes de Fine Licht, Michaela Blott, and Torsten Hoefler. 2018. Designing Scalable FPGA Architectures Using High-level Synthesis. In Proceedings of the 23rd ACM SIGPLAN Symposium on Principles and Practice of Parallel Programming (PPoPP '18). ACM, New York, NY, USA, 403-404. https://doi.org/10.1145/3178487.3178527

[6] Tom Deakin, James Price, Matt Martineau, and Simon McIntoshSmith. 2016. GPU-STREAM v2.0: Benchmarking the Achievable Memory Bandwidth of Many-Core Processors Across Diverse Parallel Programming Models. In High Performance Computing, Michela Taufer, Bernd Mohr, and Julian M. Kunkel (Eds.). Springer International Publishing, Cham, 489-507.

[7] Tobias Grosser, Albert Cohen, Justin Holewinski, P. Sadayappan, and Sven Verdoolaege. 2014. Hybrid Hexagonal/Classical Tiling for GPUs. In Proceedings of Annual IEEE/ACM International Symposium on Code Generation and Optimization (CGO '14). ACM, New York, NY, USA, Article 66, 10 pages. https://doi.org/10.1145/2581122.2544160

[8] Tobias Grosser, Albert Cohen, Paul H. J. Kelly, J. Ramanujam, P. Sadayappan, and Sven Verdoolaege. 2013. Split Tiling for GPUs: Automatic Parallelization Using Trapezoidal Tiles. In Proceedings of the 6th Workshop on General Purpose Processor Using Graphics Processing Units (GPGPU-6). ACM, New York, NY, USA, 24-31. https: //doi.org/10.1145/2458523.2458526

[9] Tobias Grosser, Sven Verdoolaege, Albert Cohen, and P. Sadayappan. 2013. The Promises of Hybrid Hexagonal/Classical Tiling for GPU. Research Report RR-8339. INRIA. https://hal.inria.fr/hal-00848691

[10] Tobias Grosser, Sven Verdoolaege, Albert Cohen, and P. Sadayappan. 2014. The Relation Between Diamond Tiling and Hexagonal Tiling. Parallel Processing Letters 24, 03 (2014), 1441002. https://doi.org/10.1142/S0129626414410023 arXiv:https://doi.org/10.1142/S0129626414410023

[11] Bastian Hagedorn, Larisa Stoltzfus, Michel Steuwer, Sergei Gorlatch, and Christophe Dubach. 2018. High Performance Stencil Code Generation with Lift. In Proceedings of the 2018 International Symposium on Code Generation and Optimization (CGO 2018). ACM, New York, NY, USA, 100-112. https://doi.org/10.1145/3168824

[12] Justin Holewinski, Louis-Noël Pouchet, and P. Sadayappan. 2012. Highperformance Code Generation for Stencil Computations on GPU Architectures. In Proceedings of the 26th ACM International Conference on Supercomputing (ICS '12). ACM, New York, NY, USA, 311-320. https://doi.org/10.1145/2304576.2304619

[13] F. Irigoin and R. Triolet. 1988. Supernode Partitioning. In Proceedings of the 15th ACM SIGPLAN-SIGACT Symposium on Principles of Programming Languages (POPL '88). ACM, New York, NY, USA, 319329. https://doi.org/10.1145/73560.73588

[14] Shoaib Kamil, Kaushik Datta, Samuel Williams, Leonid Oliker, John Shalf, and Katherine Yelick. 2006. Implicit and Explicit Optimizations for Stencil Computations. In Proceedings of the 2006 Workshop on Memory System Performance and Correctness (MSPC '06). ACM, New York, NY, USA, 51-60. https://doi.org/10.1145/1178597.1178605

[15] E. Konstantinidis and Y. Cotronis. 2016. A Quantitative Performance Evaluation of Fast on-Chip Memories of GPUs. In 2016 24th Euromicro International Conference on Parallel, Distributed, and Network-Based Processing (PDP). 448-455. https://doi.org/10.1109/PDP.2016.56

[16] Sriram Krishnamoorthy, Muthu Baskaran, Uday Bondhugula, J. Ramanujam, Atanas Rountev, and P Sadayappan. 2007. Effective Automatic Parallelization of Stencil Computations. In Proceedings of the 28th ACM SIGPLAN Conference on Programming Language Design and Implementation (PLDI '07). ACM, New York, NY, USA, 235-244. https://doi.org/10.1145/1250734.1250761

[17] Naoya Maruyama and Takayuki Aoki. 2014. Optimizing Stencil Computations for NVIDIA Kepler GPUs. In Proceedings of the 1st International Workshop on High-Performance Stencil Computations, Armin Größlinger and Harald Köstler (Eds.). Vienna, Austria, 89-95. http://www.exastencils.org/histencils/2014/

[18] Jiayuan Meng and Kevin Skadron. 2009. Performance Modeling and Automatic Ghost Zone Optimization for Iterative Stencil Loops on GPUs. In Proceedings of the 23rd International Conference on Supercomputing (ICS '09). ACM, New York, NY, USA, 256-265. https: //doi.org/10.1145/1542275.1542313

[19] Takayuki Muranushi and Junichiro Makino. 2015. Optimal Temporal Blocking for Stencil Computation. Procedia Computer Science 51 (2015), 1303 - 1312. https://doi.org/10.1016/j.procs.2015.05.315 International Conference On Computational Science, ICCS 2015.

[20] A. Nguyen, N. Satish, J. Chhugani, C. Kim, and P. Dubey. 2010. 3.5-D Blocking Optimization for Stencil Computations on Modern CPUs and GPUs. In SC '10: Proceedings of the 2010 ACM/IEEE International Conference for High Performance Computing, Networking, Storage and Analysis. 1-13. https://doi.org/10.1109/SC.2010.2

[21] Nirmal Prajapati, Waruna Ranasinghe, Sanjay Rajopadhye, Rumen Andonov, Hristo Didjev, and Tobias Grosser. 2017. Simple, Accurate, Analytical Time Modeling and Optimal Tile Size Selection for GPGPU Stencils. In Proceedings of the 22Nd ACM SIGPLAN Symposium on Principles and Practice of Parallel Programming (PPoPP '17). ACM, New York, NY, USA, 163-177. https://doi.org/10.1145/3018743.3018744

[22] Mahesh Ravishankar, Justin Holewinski, and Vinod Grover. 2015. Forma: A DSL for Image Processing Applications to Target GPUs and Multi-core CPUs. In Proceedings of the 8th Workshop on General Purpose Processing Using GPUs (GPGPU-8). ACM, New York, NY, USA, 109-120. https://doi.org/10.1145/2716282.2716290

[23] Prashant Rawat, Martin Kong, Tom Henretty, Justin Holewinski, Kevin Stock, Louis-Noël Pouchet, J. Ramanujam, Atanas Rountev, and P. Sadayappan. 2015. SDSLc: A Multi-target Domain-specific Compiler for Stencil Computations. In Proceedings of the 5th International Workshop on Domain-Specific Languages and High-Level Frameworks for High Performance Computing (WOLFHPC '15). ACM, New York, NY, USA, Article 6, 10 pages. https://doi.org/10.1145/2830018.2830025

[24] Prashant Singh Rawat, Changwan Hong, Mahesh Ravishankar, Vinod Grover, Louis-Noël Pouchet, and P. Sadayappan. 2016. Effective Resource Management for Enhancing Performance of 2D and 3D Stencils on GPUs. In Proceedings of the 9th Annual Workshop on General Purpose Processing Using Graphics Processing Unit (GPGPU '16). ACM, New York, NY, USA, 92-102. https://doi.org/10.1145/2884045.2884047

[25] Prashant Singh Rawat, Fabrice Rastello, Aravind Sukumaran-Rajam, Louis-Noël Pouchet, Atanas Rountev, and P. Sadayappan. 2018. Register Optimizations for Stencils on GPUs. In Proceedings of the 23rd ACM SIGPLAN Symposium on Principles and Practice of Parallel Programming (PPoPP '18). ACM, New York, NY, USA, 168-182. https: //doi.org/10.1145/3178487.3178500

[26] P. S. Rawat, M. Vaidya, A. Sukumaran-Rajam, M. Ravishankar, V. Grover, A. Rountev, L. Pouchet, and P. Sadayappan. 2018. DomainSpecific Optimization and Generation of High-Performance GPU Code 
for Stencil Computations. Proc. IEEE 106, 11 (Nov 2018), 1902-1920. https://doi.org/10.1109/JPROC.2018.2862896

[27] Prashant Singh Rawat, Miheer Vaidya, Aravind Sukumaran-Rajam, Atanas Rountev, Louis-Noël Pouchet, and P Sadayappan. 2019. On Optimizing Complex Stencils on GPUs. In 2019 IEEE International Parallel and Distributed Processing Symposium (IPDPS).

[28] Diego Rossinelli, Babak Hejazialhosseini, Panagiotis Hadjidoukas, Costas Bekas, Alessandro Curioni, Adam Bertsch, Scott Futral, Steffen J. Schmidt, Nikolaus A. Adams, and Petros Koumoutsakos. 2013. 11 PFLOP/s Simulations of Cloud Cavitation Collapse. In Proceedings of the International Conference on High Performance Computing, Networking, Storage and Analysis (SC '13). ACM, New York, NY, USA, Article 3, 13 pages. https://doi.org/10.1145/2503210.2504565

[29] Takashi Shimokawabe, Takayuki Aoki, Chiashi Muroi, Junichi Ishida, Kohei Kawano, Toshio Endo, Akira Nukada, Naoya Maruyama, and Satoshi Matsuoka. 2010. An 80-Fold Speedup, 15.0 TFlops Full GPU Acceleration of Non-Hydrostatic Weather Model ASUCA Production Code. In Proceedings of the 2010 ACM/IEEE International Conference for High Performance Computing, Networking, Storage and Analysis (SC '10). IEEE Computer Society, Washington, DC, USA, 1-11. https: //doi.org/10.1109/SC.2010.9

[30] Takashi Shimokawabe, Takayuki Aoki, Tomohiro Takaki, Toshio Endo, Akinori Yamanaka, Naoya Maruyama, Akira Nukada, and Satoshi Matsuoka. 2011. Peta-scale Phase-field Simulation for Dendritic Solidification on the TSUBAME 2.0 Supercomputer. In Proceedings of 2011 International Conference for High Performance Computing, Networking, Storage and Analysis (SC '11). ACM, New York, NY, USA, Article 3, 11 pages. https://doi.org/10.1145/2063384.2063388

[31] W. T. Tang, W. J. Tan, R. Krishnamoorthy, Y. W. Wong, S. Kuo, R. S. M. Goh, S. J. Turner, and W. Wong. 2013. Optimizing and Auto-Tuning Iterative Stencil Loops for GPUs with the In-Plane Method. In 2013 IEEE 27th International Symposium on Parallel and Distributed Processing. 452-462. https://doi.org/10.1109/IPDPS.2013.79

[32] Yuan Tang, Rezaul Alam Chowdhury, Bradley C. Kuszmaul, Chi-Keung Luk, and Charles E. Leiserson. 2011. The Pochoir Stencil Compiler. In Proceedings of the Twenty-third Annual ACM Symposium on Parallelism in Algorithms and Architectures (SPAA '11). ACM, New York, NY, USA, 117-128. https://doi.org/10.1145/1989493.1989508
[33] TOP500.org. 2018. November 2018| TOP500 Supercomputer Sites. https://www.top500.org/lists/2018/11/

[34] Sven Verdoolaege. 2010. isl: An Integer Set Library for the Polyhedral Model. In Mathematical Software - ICMS 2010, Komei Fukuda, Joris van der Hoeven, Michael Joswig, and Nobuki Takayama (Eds.). Springer Berlin Heidelberg, Berlin, Heidelberg, 299-302. https: //doi.org/10.1007/978-3-642-15582-6_49

[35] Sven Verdoolaege, Juan Carlos Juega, Albert Cohen, José Ignacio Gómez, Christian Tenllado, and Francky Catthoor. 2013. Polyhedral Parallel Code Generation for CUDA. ACM Trans. Archit. Code Optim. 9, 4, Article 54 (Jan. 2013), 23 pages. https://doi.org/10.1145/2400682.2400713

[36] Sven Verdoolaege and Tobias Grosser. 2012. Polyhedral Extraction Tool. In Second International Workshop on Polyhedral Compilation Techniques (IMPACT'12). Paris, France. http://impact.gforge.inria.fr/impact2012/

[37] Samuel Williams, Andrew Waterman, and David Patterson. 2009. Roofline: An Insightful Visual Performance Model for Multicore Architectures. Commun. ACM 52, 4 (April 2009), 65-76. https: //doi.org/10.1145/1498765.1498785

[38] M. Wolfe. 1989. More Iteration Space Tiling. In Proceedings of the 1989 ACM/IEEE Conference on Supercomputing (Supercomputing '89). ACM, New York, NY, USA, 655-664. https://doi.org/10.1145/76263.76337

[39] C. Yount, J. Tobin, A. Breuer, and A. Duran. 2016. YASK-Yet Another Stencil Kernel: A Framework for HPC Stencil Code-Generation and Tuning. In 2016 Sixth International Workshop on Domain-Specific Languages and High-Level Frameworks for High Performance Computing (WOLFHPC). 30-39. https://doi.org/10.1109/WOLFHPC.2016.08

[40] Hamid Reza Zohouri, Artur Podobas, and Satoshi Matsuoka. 2018. Combined Spatial and Temporal Blocking for High-Performance Stencil Computation on FPGAs Using OpenCL. In Proceedings of the 2018 ACM/SIGDA International Symposium on Field-Programmable Gate Arrays (FPGA '18). ACM, New York, NY, USA, 153-162. https: //doi.org/10.1145/3174243.3174248

[41] H. R. Zohouri, A. Podobas, and S. Matsuoka. 2018. High-Performance High-Order Stencil Computation on FPGAs Using OpenCL. In 2018 IEEE International Parallel and Distributed Processing Symposium Workshops (IPDPSW). 123-130. https://doi.org/10.1109/IPDPSW.2018. 00027 